\title{
Liver Surgery and its Capsules
}

\author{
Jacques Belghiti* and François Cauchy
}

Department of Surgery, Hospital Beaujon, University Paris 7, 92118, Clichy, France

Precise knowledge of liver anatomy is recognized as the key to a safe and efficient liver resection passing in the right surgical plan, often following hepatic veins, while preserving sufficient residual parenchyma with adequate regeneration capacity. Our knowledge of liver anatomy has evolved over centuries through numerous depictions culminating with the breakthrough description of liver segments produced by Claude Couinaud. His work was rapidly adopted and further developed by Japanese surgeons with the aim of gathering more sophisticated descriptive details in order to further refine surgical techniques. It is therefore not surprising that most novelties in the area of liver anatomy arose from Japan and thus provided immediate surgical application. With such meticulous descriptions of the vascular anatomy using 3-D CT reconstruction, one may even wonder what will remain of the classification of Couinaud for the next generation of surgeons.

For a long period of time, the liver capsule described by Francis Glisson and René-Théophile-Hyacinthe Laennec was disregarded as an anatomic structure of limited importance and the surgical impact of its extension around both Glissonean pedicles and hepatic veins was underestimated. The present issue of Surgery, Gastroenterology and Oncology disproves this tradition and provides an original surgical application of both parenchymal and vascular capsules. In view of our own surgical experience, the first description of the liver hanging was concentrated on the avascular space in front of the inferior vena cava (IVC) (1). Of course, the anatomical significance of this maneuver remained poorly explored until the outstanding publication of Atsushi Sugioka et al. as featured in this special issue. Yet, we always noticed that inaccurate placement of the clamp during the maneuver could result in substantial bleeding, which could only be controlled thanks to the pressure of the liver mass. These considerations finely highlight the description of the two layers of capsule surrounding the IVC and hepatic veins, illustrate the risk of the inner-Laennec approach, and also supports the safety of the inter-Laennec approach when the clamp is passed through the adequate plane.

Naturally, other relevant applications of this refined anatomical description are highlighted in the present issue. Among these, the one-way resection based on Laennec's capsule, which combines extrahepatic Glissonean pedicle isolation according to Takasaki's principles with the
Corresponding author: Professor Jacques Belghiti Department of Surgery Hospital Beaujon, University Paris 7 92118, Clichy, France E-mail: jacques.belghiti@aphp.fr
Copyright (ㅇ Celsius Publishing House www.sgo-iasgo.com 
"hepatic vein root-at first one-way" parenchymal dissection deserves particular attention. In an era where laparoscopic liver resection should aim at obtaining at least similar quality criteria as the open approach, the laparoscopic one-way approach certainly represents a step towards technical standardization while ensuring patient's safety.

Finally, taking into account these capsules from a technical point of view in both open and laparoscopic approaches may also have important oncologic consequences. The dissection under the capsule may represent an oncologic plane, which could explain the good, yet poorly understood, results of the "vascular R1" resection in patients undergoing hepatec- tomy for colorectal liver metastases as well as hepatocellular carcinoma, as reported by Torzilli et al. $(2,3)$.

\section{REFERENCES}

1. Belghiti J, Guevara OA, Noun R, Saldinger PF, Kianmanesh R. Liver hanging maneuver: a safe approach to right hepatectomy without liver mobilization. J Am Coll Surg. 2001;193(1):109-11.

2. Viganò L, Procopio F, Cimino MM, Donadon M, Gatti A, Costa G, et al. Is Tumor detachment from vascular structures equivalent to Ro resection in surgery for colorectal liver metastases? An observational Cohort. Ann Surg Oncol. 2016;23(4):1352-60.

3. Donadon M, Terrone A, Procopio F, Cimino M, Palmisano A, Viganò $L$, et al. Is $R 1$ vascular hepatectomy for hepatocellular carcinoma oncologically adequate? Analysis of 327 consecutive patients. Surgery. 2019;165(5):897-904. 